\title{
The Structure and Evolution of Dense Cores
}

\author{
Gary A. Fuller \\ Physics Department, UMIST, PO Box 88, Manchester, M60 1QD, UK
}

\begin{abstract}
A small fraction of the mass of a molecular cloud is in dense, gravitationally bound structures many of which eventually form stars. This paper reviews some of the important properties of these dense cores. Ideas about the formation of these coies are summarised and recent observational results on the structure and evolution of cores reviewed.
\end{abstract}

\section{Introduction}

Dense cores are the gravitationally bound parcels of gas which form stars in molecular clouds. At a density of $\sim 3 \times 10^{4} \mathrm{~cm}^{3}$, these regions range in diameter from $\sim 0.05 \mathrm{pc}$ to $0.3 \mathrm{pc}$, and in mass from $\sim 0.5 \mathrm{M} \odot$ to $\sim 100 \mathrm{M} \odot(J i j i n a$, Adams $\&$ Myers 1999). The lowest mass cores therefore contain enough mass to form only one, or at most a few stars, whereas the more massive ones could form (and indeed are forming) clusters of many stars. Despite the ongoing star formation in many cores, they are close to being in virial equilibrium with the gravitational energy balanced by the kinetic energy in the gas motions as measured from the widths of molecular lines. The linewidths always show motions in excess of thermal but in low mass cores the non-thermal motions are very small: the thermal pressure in these cores is $90 \%$ to $95 \%$ of the total pressure.

Below I discuss some of the properties of these cores and how they form and evolve.

\section{The Formation of Cores}

The formation of stars in a molecular cloud requires the formation of tightly bound substructures, dense cores, within which gravity eventually overwhelms the other forces leading to collapse and formation of a protostar. Although giant molecular clouds (GMCs) are gravitationally bound as a whole, only a few of the most massive clumps within a cloud are bound. The majority of the clumps can only be stable if they are pressure confined (Bertoldi \& McKee 1992). To become gravitationally bound, rather than pressure confined, the molecular material must lose some of the turbulent motions which is seen in their linewidths.

These turbulent motions are supersonic but sub-Alfvénic (Myers \& Goodman 1988; Myers \& Khersonsky 1995; Heyer \& Schloerb 1997; Crutcher 1998) and are believed to arise from waves on the magnetic field in the cloud. The loss of turbulence within a core can be seen in the variation of the (nonther- 
mal) linewidth of molecular transitions within a core (e.g. Fuller \& Myers 1992; Goodman et al. 1998).

Three mechanisms which may lead to the suppression of the turbulent motions have been suggested. If some molecular material becomes denser than its surroundings the enhanced dissipation rate and reduced coupling to external wave sources may lead to lower turbulence in the dense gas (e.g. Nakano 1998). Such density enhancements may occur through a random fluctuation due to the turbulent motions themselves (Elmegreen 1999) or some external cause such as a compression due to an increased external pressure or colliding gas flows. Alternatively the coupling between the magnetic field and the gas may be reduced as an increase in the extinction reduces the ionization fraction in a region (McKee 1983; Myers \& Khersonsky 1995). The third alternative is that the magnetic field which supports the turbulence may diffuse out of a region by ambipolar diffusion (e.g. Mouschovias 1979; Nakano 1979; Lizano \& Shu 1989). In any one region a combination of these effects is likely to be operating leading to the formation of bound dense cores and the start of collapse to form a star. For example, a collision between two gas flows may form cores in a critical state which a small amount of ambipolar diffusion pushes over the edge to star formation.

One difficulty with understanding core formation is that in even in the most active star forming clouds only a small fraction of the mass of the cloud is in the dense cores. For example in Taurus about $10 \%$ of the total mass of the cloud is in gas at a number density of $\sim 10^{4} \mathrm{~cm}^{-3}$ (Onishi et al. 1998). In other words the process which forms cores needs to be relatively inefficient, converting only about $10 \%$ of a cloud into dense cores at any time.

\section{Evolution to Star Formation}

Jessop \& Ward-Thompson (2000) compiled the results of a number of surveys of dense cores (including their own IRAS based survey) and investigated the incidence of IRAS point sources with the cores to estimate the typical lifetime of a dense core as a function of average density before it forms a star. Their results make it clear that denser cores form stars more rapidly. At a number density of $10^{3} \mathrm{~cm}^{-3}$ the prestellar lifetime of a core is between 7 and 9 free fall times whereas once the density has reached $10^{4} \mathrm{~cm}^{-3}$, the prestellar lifetime has dropped to only 3 free fall times. The other way to view this result is that the denser a core becomes the more likely it is to form a star.

Onishi et al. (1998) in their survey of $\mathrm{C}^{18} \mathrm{O}$ dense cores in Taurus found a threshold column density for star formation of $8 \times 10^{21} \mathrm{~cm}^{-2}$. This threshold shows that the evolution of dense cores to the point of star formation involves an evolution in the structure of the whole core over a relatively large region several times $0.1 \mathrm{pc}$ in size, not just a small central region within a core.

\subsection{Density Profile}

There has been much effort expended measuring the density profile in cores around embedded sources and pre-stellar cores as the density profiles reflect how the gas is supported and can potentially identify the regions which are infalling through their characteristic $r^{-3 / 2}$ profile. The density profiles also provide a picture of the mass accretion rate as the core collapses. 
The inner, $r<10000 \mathrm{AU}$, regions of cores around embedded sources have relatively steep profiles in the range $r^{-1.5}$ to $r^{-2}$, even for the youngest sources (eg. Chandler \& Richer 2000; Shirley et al. 2000). Apparent departures from these steep profiles more likely reflect unaccounted for heating from the outflow from the source rather than true changes in the density profile. In the outer regions of the cores, where non-thermal motions are more significant, the density profiles flatten to closer to $r^{-1}$ (Myers \& Fuller 1992).

Observations show that starless, or pre-stellar, cores have steep density profiles in their envelopes similar to the cores with embedded sources (WardThompson, Motte \& André 1999). However, this profile flattens in the central regions becoming nearly constant density within $0.01 \mathrm{pc}$ to $0.04 \mathrm{pc}$ of the core centre. At present it is unclear how the starless cores evolve to star formation. If they collapse from this flat centre state, then the flat central density profile results in an initial burst of high mass accretion followed by a declining infall rate (e.g. Hendriksen, André, \& Bontemps 1997). On the other hand it has been argued that the inner regions of the starless cores quasi-statically evolve toward steeper density profiles approaching the $r^{-2}$ profile of a singular isothermal sphere before becoming unstable and undergoing dynamical collapse ( $\mathrm{Li} \&$ Shu 1996).

An important issue about dense cores is whether they have an outer boundary (or a characteristic size scale) which would imply that these cores are effectively forming stars from a fixed mass reservoir or whether cores smoothly connect to larger scale structures. Isolated cores such as those in Bok globules clearly do have a limited amount of material out of which stars can form, although this is many times larger than the mass of the stars forming (or likely to form) in the region. An absorption study of 24 cores by Bacmann et al. (2000) found that the cores reach a density compared to the background value at distances between $0.05 \mathrm{pc}$ and $0.3 \mathrm{pc}$ from their centres, a range of sizes which encompasses a wide range of mass. These sizes are similar to the velocity coherence size scale, where the nonthermal motions of the gas rapidly decrease, identified by Goodman et al. (1998). Only three or four cores in the Bacmann et al. sample showed sharp outer edges. However in some regions the cores do appear to have a characteristic size. Recent high resolution observations of the $\rho$ Oph cloud by Motte et al. (1998) found that the dense gas in this region has a characteristic size scale of about $0.024 \mathrm{pc}$. It is not yet known why some regions contain cores of a much more limited range of sizes than other regions and why some cores have very sharp outer edges while others blend smoothly into their parent clouds.

\subsection{Mass Distribution of Cores}

The mass spectrum of clumps in different molecular clouds is remarkably consistent with $d N \propto M^{-p} d M$ with $p=1.5 \rightarrow 1.7$ which is much flatter than the high mass end of the stellar initial mass function which has slope $p \sim 2.7$ for stars with mass in the range $1 \mathrm{M}_{\odot} \leq M_{*} \leq 10 \mathrm{M}_{\odot}$ (e.g. Scalo 1998). However, as discussed above, the clumps in clouds are not the structures which are forming stars. Recent studies of the small scale structure in clouds have shown the high density components of dense cores have much steeper mass distributions with $p \sim 2.5$ (Motte et al. 1998; Testi \& Sargent 1998; Onishi et al. 1999) for cores 
with masses greater than $0.5 \mathrm{M}_{\odot}$ with a flatter slope for lower mass cores, very similar to the IMF. These observations suggest that it is this mass distribution of cores which directly determines the stellar IMF. However, this requires that the masses of starless cores do not increase before they collapse to form stars and that all cores form stars with the same efficiency.

\subsection{Core Shapes}

A number surveys have found dense cores to have of mean projected axial ratios of about 2:1 (e.g. Myers et al. 1991; Bourke et al. 1995). Comparison of this value with that expected from a random distribution of fixed axial ratio prolate or oblate spheroids lead Myers et al. (1991) to conclude that cores are probably intrinsically prolate, especially when different tracers show a well aligned shape over a wide range of size scales. A similar conclusion was reached by Ryden (1996) although she also concluded that the sample of objects in her analysis probably did not represent projections of a randomly oriented distribution. The origin of the flattening is unclear but the lack of a correlation between the rotation axes and the minor axes of the cores indicates that the cores are not rotationally flattened (Goodman et al. 1993).

In addition to the shapes of dense cores, observations of the circumstellar environment of young sources point towards the dense gas out of which protostars form being quite flattened. There are a number of Class 0 , or very young Class I, sources which are now known to be surrounded by relatively large, $r \sim 10000$ AU, flattened dense structures. Examples include HH211 (Wiseman, Fuller \& Wootten 2000) and L483 (Fuller \& Wootten 2000; Fig. 1). The highly collimated outflows from such sources make it difficult to interpret these structures being the remnants of much more spherically symmetric structures as has been suggested in the past. If common around very young sources, these structures point to the fact that the stars form from a very flattened distribution of dense gas. Modelling of the reflection nebulae associated with many young sources also suggests that these objects form in flattened cores (Hartmann, Calvet \& Boss 1996).

\subsection{Observations of Infall}

Since Zhou et al. (1993) claimed the spectroscopic detection of gravitational infall of material around B335 based on the line asymmetries observed in CS and $\mathrm{H}_{2} \mathrm{CO}$, similar line asymmetries have been detected towards several other young sources (e.g. Mardones et al. 1997; Gregersen et al. 1997). For those sources which have been modelled in detail, the line profiles have typically been modelled with singular isothermal sphere (SIS) collapse models to derive the mass accretion rate and infall radius. These studies find typical infall velocities of order $0.1 \mathrm{~km} / \mathrm{s}$ up to about $0.6 \mathrm{~km} / \mathrm{s}$ (Mardones et al. 1997) and outer radii of the infalling material of about $0.03 \mathrm{pc}$ to $0.1 \mathrm{pc}$. The implied mass accretion rates are typically a few times $10^{-6} \mathrm{M}_{\odot} / \mathrm{yr}$ up to $5 \times 10^{-5} \mathrm{M}_{\odot} / \mathrm{yr}$. The large infall velocities and hence mass accretion rates occur for sources which have line profiles with large nonthermal (turbulent) components, not necessarily the youngest (or indeed oldest) sources. Too few sources have yet been modelled in detail to draw many firm conclusions about the variation of the accretion properties with source age, although comparsion of the infall line asymmetries 

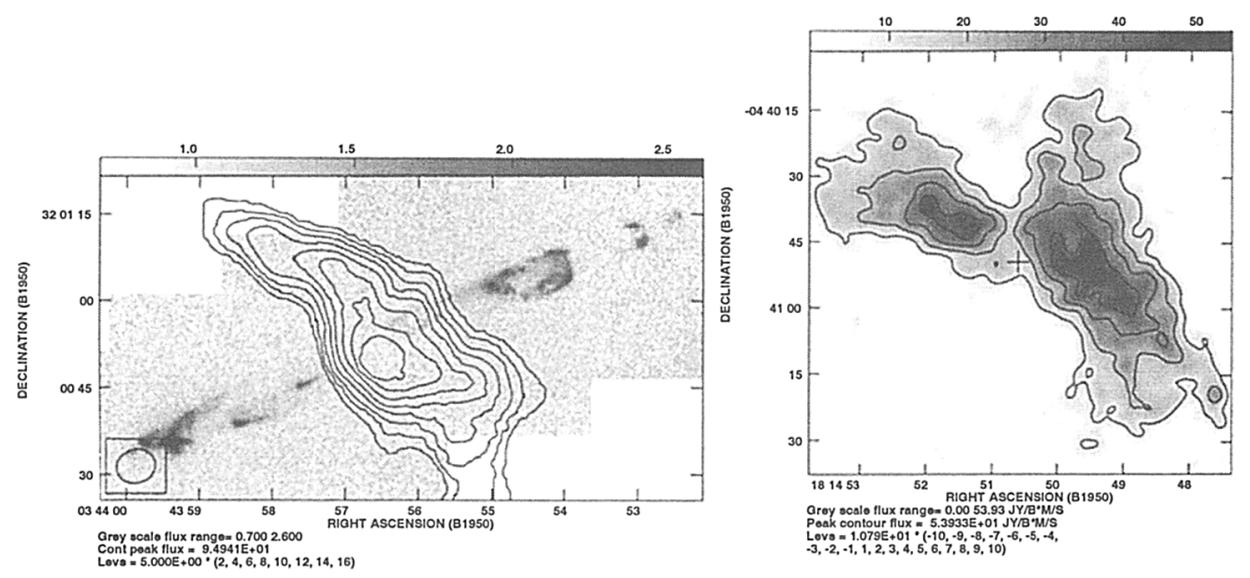

Figure 1. Images of integrated ammonia $(1,1)$ emission measured at the VLA towards the very young sources HH211 (left) and L483 (right). The grayscale in the HH211 image shows the highly collimated molecular hydrogen jet driven by the embedded protostar (McCaughrean, Rayner \& Zinnecker 1994).

observed towards Class 0 and Class I sources does not show any evidence that the Class 0 to Class I transition marks the end of the infall stage (Gregersen et al. 2000). On the other hand indirect evidence from outflows does suggest a decrease in accretion as sources evolve.

Models of bipolar outflow generation predict a direct proportionality between mass accretion and mass outflow rates (Shu et al. 1994; Fiege \& Henriksen 1996; Ouyed \& Pudritz 1997), so the observed mass outflow rates can be used to infer accretion rates. The decrease in mass outflow rate from Class 0 to Class I sources measured by Bontemps et al. (1996) implies a decrease in mass accretion rate from $\sim 10^{-5} \mathrm{M}_{\odot} / \mathrm{yr}$ for Class 0 sources to $\sim 2 \times 10^{-7} \mathrm{M}_{\odot} / \mathrm{yr}$ for the most evolved Class I sources. However it should be borne in mind that the outflow mass loss rate is proportional to the accretion rate through the circumstellar disk, which at a given moment is not necessarily directly related to the mass accretion rate in the dense core envelope to which the observations of Gregersen et al. were sensitive.

One important point to keep in mind is that only $23 \%$ of the young sources in the Gergersen et al. (2000) survey showed line asymmetries indicating infall, even though it is expected that at least all the Class 0 sources are undergoing significant accretion. In addition, towards many sources different molecular tracers have different line profiles. In the most extreme cases some tracers show the infall asymmetry whereas others have reverse asymmetry, clearly indicating that our models of infall are currently far too simplistic to interpreting increasing complete observations. As an example consider the protostar in L483. 

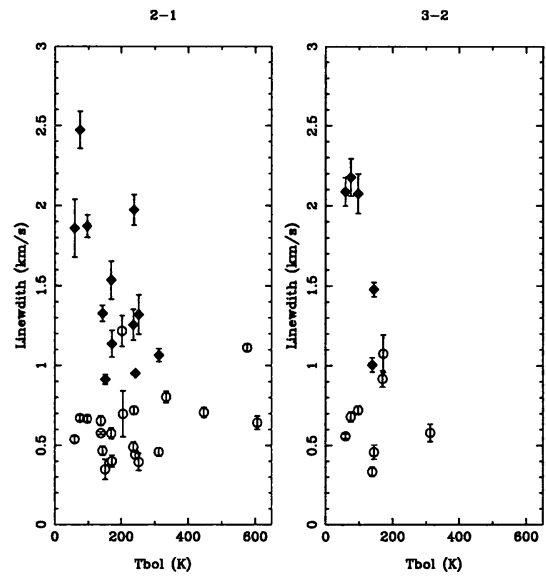

Figure 2. Linewidth of $\mathrm{C}^{18} \mathrm{O}$ versus bolometric temperature for a nearly complete sample of Class 0 and I sources in Taurus. For sources with two velocity components, the full width half maximum width of the broad component is shown as a solid symbol. The left panel shows the results for the $J=2-1$ transition and the right for the $J=3-$ 2 transition. Notice how the broad components are broader for the younger (lower bolometric temperature) sources.

Observations of $\mathrm{H}_{2} \mathrm{CO}$ lines towards this source by Myers et al. (1995) suggested that the envelope around the star was infalling and Park, Jongsoo \& Minh (1999) modelled the HCN emission with an SIS infall model and found that infall was occuring within the $0.1 \mathrm{pc}$ size region around the central star. Higher angular resolution observations of the $\mathrm{NH}_{3}$ emission by Fuller \& Wootten (2000) show the $\mathrm{NH}_{3}(1,1)$ and $(2,2)$ transitions to have linewidths and velocities consistent with infall, but are difficult to model in detail with a simple SIS infall model. Also the filament in which the protostar is forming has a transverse dimension considerably smaller than the infall radius deduced by Park et al. (1999).

Clearly further progress on understanding infall will require much more complete models which include the initially flattened distribution of material and the effects of the outflow on the circumstellar material.

\subsection{Angular Momentum}

One of the important parameters which controls the collapse and fragmentation of dense cores is their angular momentum and its evolution during collapse. Models of core collapse are able to follow the angular momentum distribution during the collapse (e.g. Tomisaka 2000, and this volume), but only little is known observationally.

Goodman et al. (1993) presented an analysis of velocity gradients detected in $\mathrm{NH}_{3}$ cores and showed that most cores do have measurable velocity gradients, although they are not energetically important on the $0.1 \mathrm{pc}$ size scale of the ammonia cores (see also Barranco \& Goodman 1998). The ratio of rotational to 
gravitational energy in the ammonia cores was $\sim 0.02$ and the typical specific angular momentum a few times $10^{21} \mathrm{~cm}^{2} \mathrm{~s}^{-1}$. Simon et al. (1995) showed that the tail of core specfic angular momentum distribution stretches into the range of the angular momentum of the binary stars in Taurus showing that at least some of the cores which form binary systems have already solved their angular momentum problem by the time they have reached a density of $\sim 3 \times 10^{4} \mathrm{~cm}^{-3}$.

Goodman et al. also found a correlation of velocity gradient with core size. Note however that this correlation was found in a sample of cores of different sizes all observed in the same molecular transition. In the nomenclature of the Goodman et al. (1998) discussion of linewidth-size correlations this would be a Type-2 correlation. This correlation has little, if anything, to do with how the velocity gradient and angular momentum vary with radius within a given core (a type 3 or 4 correlation) which is the quantity of interest for comparison with collapse and evolution models. Understanding this requires imaging and analysis of cores over a range of size scales from the outer reaches of the cores down to the dense gas in the circumstellar regions and the comparison of the velocity structure of the circumstellar regions of Class 0 and Class I sources (e.g. Wiseman, Fuller \& Wootten 2000). Some work looking at the angular momentum of the inner regions of cores has been done by Ohashi et al. (1997) who found a suggestion that the inner $6000 \mathrm{AU}$ radius regions of cores have relatively constant angular momentum.

\section{Dispersal of Cores}

Young T Tauri stars which are no longer intimately associated with dense molecular gas show that on time scales of about a million years the dense core gas out of which these stars formed has dissipated. From an analysis of the column density of gas towards the Class 0 and Class I sources in Taurus Ladd, Fuller \& Deane (1999) showed that the clearing rate is a function of source age being fastest for the youngest sources. Whether the bulk of the material either infalls on to the central star or is dispersed is unclear. Dispersal could occur by the material infalling to the central parts of the disk and then being ejected, although this appears at odds with outflow generation models which show that only some fraction of the material passing through the disk is ejected. Alternatively the material could be directly cleared through the action of the outflow from the star or as a result of becoming unbound as mass is removed by the outflow from the inner regions of the core.

Detailed analysis of the $\mathrm{C}^{18} \mathrm{O}$ and $\mathrm{C}^{17} \mathrm{O}$ line profiles towards this Taurus sample shows that the lines are composed of a velocity component 0.5 to 0.7 $\mathrm{km} / \mathrm{s}$ wide plus a broader component. The width of this broader component is largest for the youngest sources (Figure 2; Fuller \& Ladd 2000) and is most likely a component of the outflow from the sources. The decrease in velocity width with increasing source age could reflect either decreasing outflow and wind power as sources evolve or a decrease in the coupling of the stellar wind to the circumstellar material.

The broad components contain a substantial fraction, $20 \%$ to $50 \%$, of the mass in the beam showing that even for the very youngest sources the outflow is having a profound effect on the circumstellar material and that this broad 
component can clear the circumstellar region rapidly enough to account for the observed decrease in column density. In contrast to the youngest sources, the absence of broad components towards older sources suggests that outflows are unimportant in clearing the circumstellar regions of sources past the early Class I phase, suggesting that the bulk of the circumstellar material (except perhaps in the disks) has already been removed by this phase in a protostar's evolution. The rapid clearing of the circumstellar material means that the mass of the star (or stars) formed is fixed very early during the Class 0/early Class I phase in the evolution of the star.

\section{Conclusions and Implications for Binary Formation}

The very young embedded binary/multiple protostar systems now known (Mun$\mathrm{dy}$, this volume) are most easily explained as the results of fragmentation during collapse. Numerical models of collapsing cores find that fragmentation occurs in rapidly rotating, slowly collapsing cores with shallow density gradients with the cores initially far from equilibrium (e.g. Boss 1999; Myhill \& Kaula 1992). This is somewhat at odds with the observed properties of cores which show cores to evolve rapidly, rotate slowly and are close to equilibrium throughout their evolution and, in at least their outer regions, have relatively steep density profiles.

Fragmentation calculations therefore seem to imply that it is the collapse of the constant density central regions of starless cores which forms protobinary systems, rather than cores that evolve to steeper density profiles before collapsing. However, cores with steep density profiles may be able to fragment if there are initial density fluctuations which seed binary formation, imprinted on the cores from the decay of turbulence (Klein, this volume). If these fluctuations are even as large as $30 \%$ to $40 \%$ of the local density they would probably not yet have been recognised observationally.

In summary while it is becoming increasing clear that many binary systems are formed during the early formation and collapse of dense cores, we seem to still have some way to go to understand the parameters which govern the fragmentation and subsequent evolution. Observationally we need more complete studies of the properties of dense cores to get better estimates of the true distribution of parameters. Also studies which trace the properties of cores both to smaller interior scales and connect the core with its larger scale surroundings are needed. More detailed and realistic modelling of infall of flattened cores, including the role of outflow interaction, will help refine our understanding of the accretion process and perhaps explain why such a small fraction of the youngest sources show spectroscopic evidence of infall in their envelopes.

\section{References}

Barranco, J. A., \& Goodman, A. A. 1998, ApJ, 504, 207

Bertoldi, F., \& McKee, C. F. 1992, ApJ, 395, 140

Bontemps, S., André, P., Terebey, S., \& Cabrit, S. 1996, A\&A, 311, 858 
Bourke, T. L., Hyland, A. R., Robinson, G., James, S. D., \& Wright, C. M. 1995, MNRAS, 276, 1067

Boss, A. P. 1994, ApJ, 520, 744

Burkert, A., \& Bodenheimer, P., 2000, ApJ, in press

Chandler, C. J., \& Richer, J. R. 2000, ApJ, 530, 851

Crutcher, R. M. 1998, in Interstellar Turbulence, Proc. 2nd Guillermo Haro Conf., ed. J. Franco \& A. Carraminana, Cambridge Univ. Press

Elmegreen, B. 1999, ApJ, 527, 266

Fiege, J. D., \& Henriksen, R. N. 1996, MNRAS, 281, 1038

Fuller, G. A., \& Myers, P. C. 1992, ApJ, 348, 523

Fuller, G. A., \& Wootten, A. 2000, ApJ, 534, 854

Fuller, G. A., \& Ladd, E. F. 2000, in preparation

Gregersen, E. M., Evans, N. J., Zhou, S., \& Choi, M. 1997, ApJ, 484, 256

Goodman, A. A., Benson, P. J., Fuller, G. A., \& Myers, P. C. 1993, ApJ, 406, 528

Goodman, A. A., Barranco, J. A., Wilner, D. J., \& Heyer, M. H. 1998, ApJ, 504,223

Hartmann, L., Calvet, N., \& Boss, A. 1996, ApJ, 464, 387

Heyer, M. H., \& Schloerb, F. P., 1997, ApJ, 475, 173

Hendriksen, R. N., André, P., \& Bontemps, S. 1997, A\&A, 323, 549

Jessop, N., \& Ward-Thompson, D. 2000, MNRAS, 311, 63

Jijina, J., Adams, F. C., \& Myers, P. C. 1999, ApJS, 125, 161

Li, Z-Y., \& Shu, F. H. 1996, ApJ, 472, 211

Lizano S., \& Shu, F. H. 1989, ApJ, 342, 834

Mardones, D., Myers, P. C., Tafalla, M., Wilner, D. J., Bachiller, R., \& Garay, G. 1997, ApJ, 489, 719

McCaughrean, M. J., Rayner, J. T., Zinnecker, H. 1994, ApJ, 436, 189

McKee, C. 1983, ApJ, 345, 782

Motte, F., André, P., \& Neri, R. 1998, A\&A, 336, 150

Mouschovias, T. Ch. 1979, ApJ, 228, 475

Myers, P. C., \& Goodman, A. A. 1988, ApJ, 326, L27

Myers, P. C., Fuller, G. A., Goodman, A. A., \& Benson, P. J. 1991, ApJ, 376, 561

Myers, P. C., Bachiller, R., Caselli, P., Fuller, G. A., Mardones, D., Tafalla, M., \& Wilner, D. J. 1995, ApJ, 449, 65

Myers, P. C., \& Khersonsky, V. K. 1995, ApJ442, 186

Myers, P. C., \& Fuller, G. A. 1992, ApJ, 396, 631

Myhill, E. A., \& Kaula, W. M. 1992, ApJ, 386, 578

Nakano, T. 1979, PASJ, 31, 697

Nakano, T. 1998, ApJ, 494, 587

Ohashi, N., Hayashi, M., Ho, P. T. P., Momose, M., Tamura, M., Hirano, N., \& Sargent, A. I. 1997, ApJ, 488, 317 
Onishi, T., Mizuno, A., Kawamura, A., \& Fukui, Y. 1999, in Star Formation 1999 , ed. T. Nakamoto

Onishi, T., Mizuno, A., Kawamura, A., Ogawa, H., \& Fukui, Y. 1998, ApJ, 502, 296

Ouyed, R., \& Pudritz, R. E. 1997, ApJ, 482, 712

Park, Y.-S., Jongsoo, K., Minh, Y. C. 1999, ApJ, 520, 223

Scalo, J. 1998, in The Stellar Initial Mass Function, ed. G. Gilmore \& D. Howell, ASP Conf. Series, 42, 201

Shirley, Y. L., Evans, N. J., Rawlings, J. M. C., \& Gregersen, E. M. 2000, ApJS, in press

Simon, M., Ghez, A. M., Leinert, Ch., Cassar, L., Chen, W. P., Howell, R. R., Jameson, R. F., Matthews, K., Neugebauer, G., \& Richichi, A. 1995, ApJ, 443, 625

Shu, F. H., Najita, J., Ostriker, E, Wilkin, F., Ruden, S., \& Lizano, S. 1994, ApJ, 429, 781

Shu, F. H., Adams, F. C., \& Lizano, S. 1987, ARA\&A, 25, 23

Testi, L., \& Sargent, A. I. 1998, ApJ, 508, L91

Tomisaka, K. 2000, ApJ, 528, L41

Ward-Thompson, D., Motte, F., \& André, P. 1999, MNRAS, 305, 143

Wiseman, J., Fuller, G. A., \& Wootten, A. 2000, in preparation.

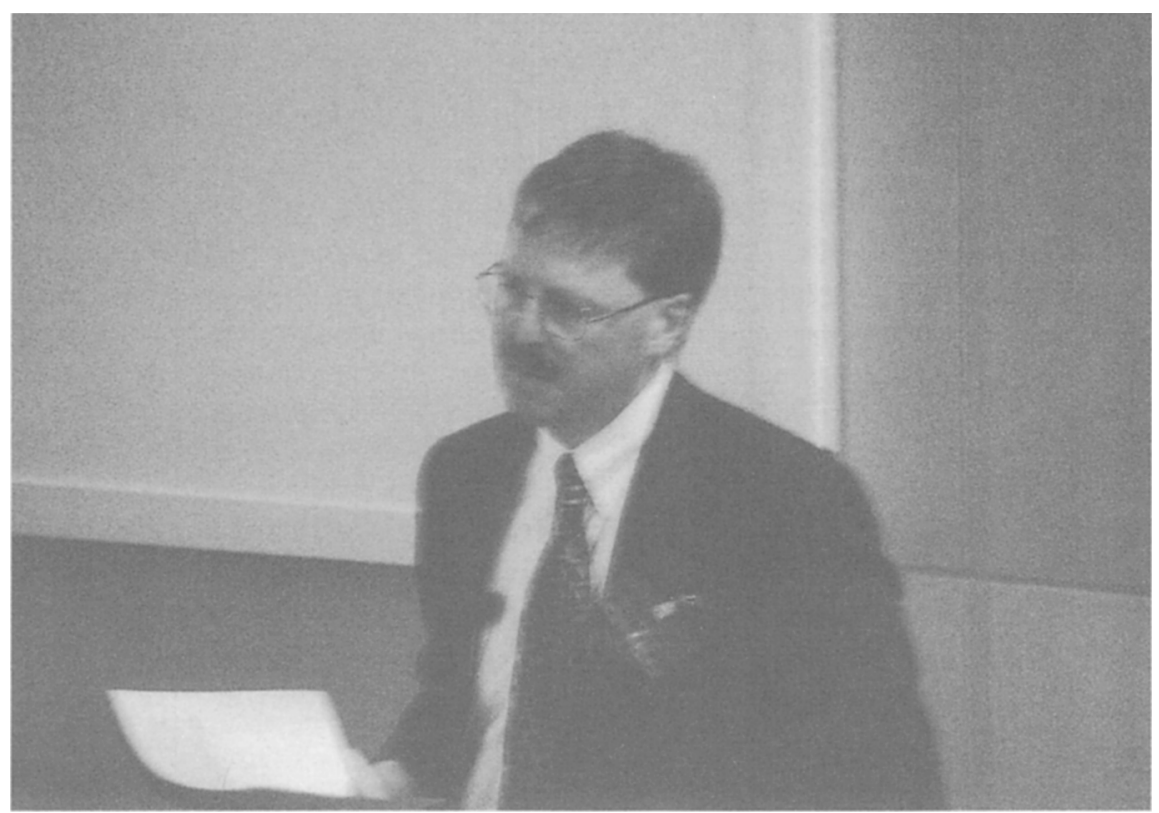

Gary A. Fuller 